\title{
ESTUDO SOBRE A TEMPERATURA DE LIQUEFAÇÃO E UTILIZAÇÃO DE MICRO-ONDAS NO PROCESSO DE HIDRÓLISE DO AMIDO DE BATATA-DOCE PARA A PRODUÇÃO DE ETANOL
}

\author{
C. M. SCHWEINBERGER ${ }^{1}$, L. F. TRIERWEILER ${ }^{1}$, T. R. PUTTI ${ }^{1}$, P. R. A. SERAFINI ${ }^{1}$, G. \\ LORENZI $^{1}$ e J. O. TRIERWEILER ${ }^{1}$
}

${ }^{1}$ Grupo de Intensificação, Modelagem, Simulação, Controle e Otimização de Processos (GIMSCOP)

Departamento de Engenharia Química, Universidade Federal do Rio Grande do Sul (UFRGS)

E-mail para contato: \{Crisms, Luciane, Jorge\} @ enq.ufrgs.br

\begin{abstract}
RESUMO - A produção de etanol no Rio Grande do Sul é inexpressiva diante do seu consumo, fato este que se deve, principalmente, à inaptidão do clima gaúcho para a produção de cana-de-açúcar. Diante desta realidade, há um mercado oportuno para matérias-primas alternativas. A batata-doce foi selecionada para o estudo devido ao seu elevado potencial de produtividade agroindustrial. Experimentos foram realizados em etapas. Primeiramente, avaliou-se o desempenho da hidrólise do amido, sob diferentes temperaturas, na etapa de liquefação, sendo constatado o melhor resultado na temperatura de $85^{\circ} \mathrm{C}$. Na segunda etapa, empregou-se o aquecimento prévio com micro-ondas, a temperatura de $85^{\circ} \mathrm{C}$ serviu como referência. Mediante os resultados de hidrólise e fermentação, concluiu-se que a inclusão da etapa de aquecimento por micro-ondas é bastante promissora, já que o tempo de aquecimento é rápido (2,5 minutos) e a temperatura de liquefação pode ser reduzida.
\end{abstract}

\section{INTRODUÇÃO}

Conforme os dados disponíveis em Unica (2014), em 2012 o Rio Grande do Sul produziu 7 milhões de litros de etanol hidratado combustível (EHC), enquanto o Estado consumiu 115 milhões de litros de EHC e 616 milhões de litros de etanol anidro. A baixa produção gaúcha se deve à inaptidão do clima para a produção de cana-de-açúcar. Diante de tal demanda há um mercado oportuno para matérias-primas alternativas.

Masiero (2012) estudou a viabilidade de microusinas de etanol (1000 L.dia $\left.{ }^{-1}\right)$ no Rio Grande do Sul envolvendo quatro matérias-primas: cana-de-açúcar, sorgo sacarino, mandioca e batata-doce. A pesquisa abrangeu o levantamento de dados teóricos de produtividade agrícola (t.ha ${ }^{-1}$ ), concentração de açúcares, concentração de amido nas matérias-primas amiláceas e custos. Após a análise dos indicadores financeiros Valor Presente Líquido (VPL), Taxa Interna de Retorno (TIR), Tempo de Retorno do Investimento e, ponderando a produtividade média dessas matérias-primas no Estado, Masiero (2012) concluiu que o sorgo sacarino e a batata-doce eram as matérias-primas viáveis.

Como o processo de produção a partir do sorgo sacarino já se encontra mais maduro, no grupo GIMSCOP do Departamento de Engenharia Química da UFRGS foram iniciados experimentos de produção de etanol a partir de batata-doce. Masiero (2012) realizou seus ensaios utilizando a enzima 
amilolítica Stargen 002, produzida pela Genencor ${ }^{\circledR}$ e desenvolvida para atuar sobre o amido na forma granular, mas, sob a recomendação de um pré-tratamento em temperaturas entre $49-63^{\circ} \mathrm{C}$. Masiero (2012) realizou o pré-tratamento de $1 \mathrm{~h}$ a $52^{\circ} \mathrm{C}$, porém para a obtenção de uma eficiência satisfatória de hidrólise, foi necessária uma elevada quantidade de enzima $(26,2 \mu \mathrm{L} / \mathrm{g}$ de batata in natura), inviabilizando o processo financeiramente.

Risso (2014) deu sequência ao trabalho de Masiero (2012), concentrando sua pesquisa no préprocessamento da matéria-prima, onde o método selecionado foi a secagem da batata, utilizando micro-ondas e posterior moagem para a obtenção de uma farinha. A aplicação de energia em temperaturas acima de $90{ }^{\circ} \mathrm{C}$, sob a presença de água, torna o amido mais suscetível a hidrólise devido à sua completa gelatinização, o que ocorre nas condições de secagem. Contudo, os resultados de Risso (2014) indicaram que há distinção na eficiência de hidrólise de acordo com o método de aquecimento. Mediante imagens obtidas com microscopia eletrônica de varredura, o método de secagem por micro-ondas demonstrou ser mais agressivo aos grânulos de amido, quando comparado ao aquecimento em estufa a $105^{\circ} \mathrm{C}$ ou solar, como pode ser visualizado na Figura 1. Observa-se nas imagens da batata seca em micro-ondas e também do aquecimento solar que a estrutura esférica original do amido sofreu maior descaracterização, sendo um possível efeito causado pela energia eletromagnética. Com seu método, Risso (2014) conseguiu baixar a dosagem da enzima Stargen 002 para $1 \mu \mathrm{L} / \mathrm{g}$ de batata in natura, o que significa uma redução de $96 \%$ em relação à dosagem utilizada por Masiero (2012).

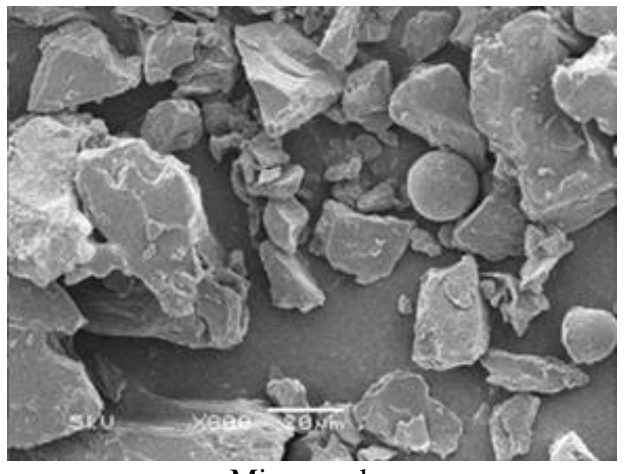

Micro-ondas

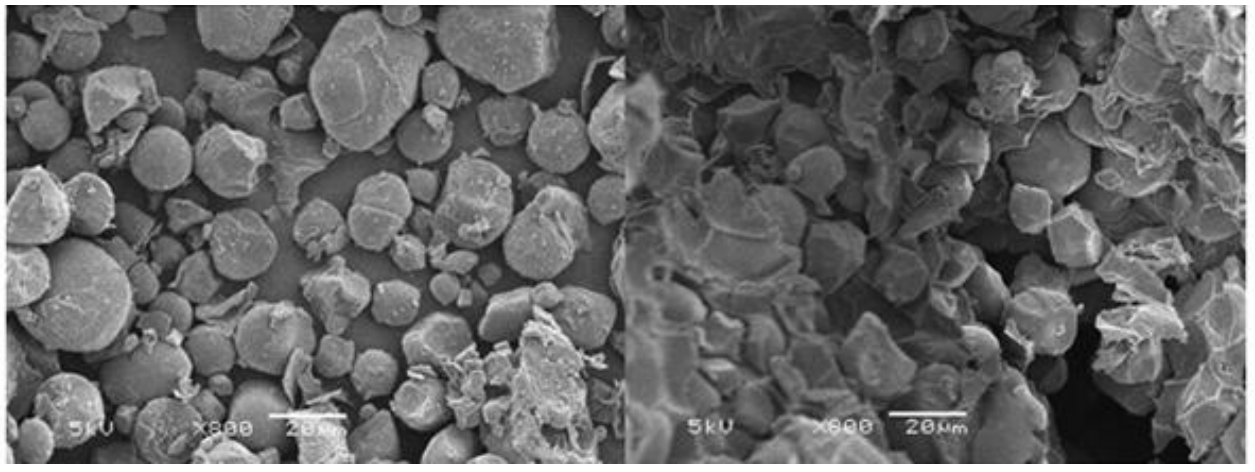

Estufa a $105^{\circ} \mathrm{C}$

Secador solar

Figura 1 - Grânulos de amido após processos de secagem. Fonte Risso (2014). 


\section{9 a 22 de outubro de 2014 \\ Florianópolis/SC}

No presente trabalho, os experimentos foram realizados com a batata-doce in natura e enzimas diferentes da Stargen 002, mas a dosagem enzimática foi na mesma concentração utilizada por Risso (2014). Foram utilizadas enzimas com temperaturas ótimas de operação superiores à recomendada pelo fabricante para a Stargen 002. Primeiramente, foram feitos experimentos variando a temperatura na liquefação, empregando métodos convencionais de aquecimento: ar quente em agitador rotativo ou água quente em banho-maria. Em um segundo momento, foram realizados experimentos utilizando micro-ondas para um aquecimento prévio, com o objetivo de reduzir a temperatura na etapa de liquefação.

\section{METODOLOGIA}

\subsection{Métodos analíticos}

A batata foi caracterizada, medindo-se o teor de umidade, glicose, frutose e glicose total, que consiste na glicose livre somada com a proveniente do amido. $\mathrm{O}$ teor de umidade foi determinado por método gravimétrico em estufa a $105^{\circ} \mathrm{C}$. Os monossacarídeos, glicose e frutose, foram determinados após a extração com álcool, onde aproximadamente $15 \mathrm{~g}$ de batata junto com $100 \mathrm{~mL}$ de álcool $70 \%$ e $0,5 \mathrm{~g}$ de carbonato de cálcio foram aquecidos em banho-maria a $85^{\circ} \mathrm{C}$ durante $1 \mathrm{~h}$. Na determinação de glicose total, aproximadamente $2 \mathrm{~g}$ de batata fresca triturada junto com $25 \mathrm{~mL}$ de água destilada e 1 $\mathrm{mL}$ de ácido clorídrico foram aquecidos em autoclave a $1 \mathrm{~atm}$ durante $2 \mathrm{~h}$. Os métodos descritos foram baseados no que consta em Figueiredo (2008). Nesta caracterização, três ou mais batatas foram trituradas em processador doméstico. O conteúdo foi homogeneizado para a amostragem e os experimentos foram realizados em triplicata. Este procedimento foi feito em três períodos, caracterizando batatas em tempo de colheitas diferentes. As amostras de hidrólise enzimática e fermentação foram centrifugadas a $5241 \mathrm{~g}$ durante 30 minutos e o sobrenadante foi analisado. A determinação de açúcares e etanol foi efetuada através de cromatografia líquida de alta eficiência (HPLC), com a coluna Hi-Plex H da Agilent, tendo como fase móvel água. A temperatura da coluna foi de $60^{\circ} \mathrm{C}$, o detector utilizado foi RID (Refractive Index Detector), a vazão da fase móvel foi de 0,6 $\mathrm{ml} / \mathrm{min}$ e volume de amostra injetado foi de $20 \mu \mathrm{L}$.

\subsection{Estudo sobre o efeito da temperatura de liquefação na hidrólise}

Neste estudo foi quantificada a produção de glicose em experimentos submetidos a diferentes temperaturas de liquefação. Foram testadas duas enzimas comerciais de liquefação: Spezyme RSL e GC 626, ambas produzidas pela Genencor ${ }^{\circledR}$. Segundo o fabricante, a Spezyme RSL possui temperatura ótima entre $83-88^{\circ} \mathrm{C}$ e $\mathrm{pH}$ ótimo entre $5-5,3$; a GC 626 possui temperatura ótima em torno de $62^{\circ} \mathrm{C}$ e pH ótimo entre 3,5 - 4. Em todos os experimentos as enzimas de liquefação foram adicionadas na mesma dosagem: $0,25 \mu \mathrm{L} / \mathrm{g}$ de batata e o tempo da operação foi de $1 \mathrm{~h}$. Após a etapa de liquefação, iniciou-se a sacarificação utilizando a enzima Distillase SSF, também produzida pela Genencor $^{\circledR}$, sendo a dosagem desta enzima de $0,75 \mu \mathrm{L} / \mathrm{g}$ de batata. A sacarificação foi realizada no pH 4,5 a $32{ }^{\circ} \mathrm{C}$ durante 24 h. A Figura 2 apresenta uma representação esquemática dos experimentos realizados.

Como consta na Figura 2, previamente às etapas de liquefação e sacarificação, foi realizada uma 
etapa de redução de viscosidade, que consistiu em submeter os meios à ação da enzima Pectinex Ultra AFP, produzida pela Novozymes ${ }^{\circledR}$ para atuar sobre substâncias pécticas. Ensaios preliminares demonstraram que a utilização de pectinase é recomendável quando se utiliza uma elevada concentração de batata, isto é, para razão massa de batata[kg]:volume de água[L] de diluição igual ou superior a 1,5:1. Trabalhar nestas concentrações é necessário para a obtenção de um vinho com a concentração de etanol igual ou superior a $10 \%{\mathrm{~V} \cdot \mathrm{v}^{-1}}^{-1}$.

\section{Experimento 1}
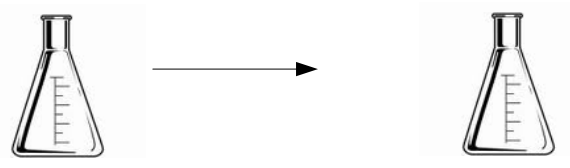

Spezyme RSL $0,25 \mu \mathrm{L} / \mathrm{g}$ batata $85^{\circ} \mathrm{C}$

Experimento 2

$\mathrm{pH} 5$
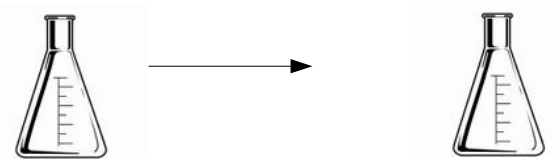

Spezyme RSL $0,25 \mu \mathrm{L} / \mathrm{g}$ batata

$70{ }^{\circ} \mathrm{C}$

Experimento 3

$\mathrm{pH} 5$
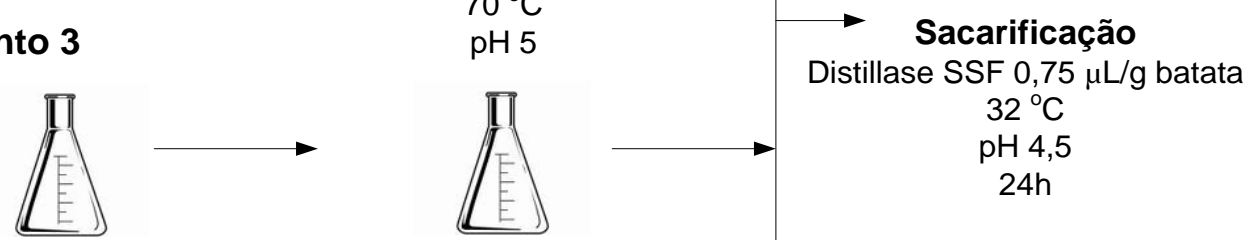

GC $6260,12 \mu \mathrm{L} / \mathrm{g}$ batata

Experimento 4

Spezyme RSL $0,13 \mu \mathrm{L} / \mathrm{g}$ batata

$70{ }^{\circ} \mathrm{C}$

$\mathrm{pH} 5$

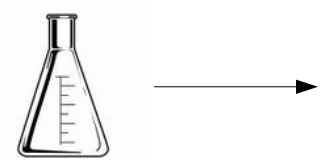

GC $6260,25 \mu \mathrm{L} / \mathrm{g}$ batata

Etapa de redução da viscosidade

Pectinex Ultra AFP 0,15 $\mu \mathrm{L} / \mathrm{g}$ batata

$40{ }^{\circ} \mathrm{C}$

$\mathrm{pH} 4$

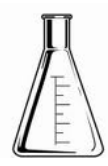

$60{ }^{\circ} \mathrm{C}$

$\mathrm{pH} 4$

$32{ }^{\circ} \mathrm{C}$

$\mathrm{pH} 4,5$

$24 \mathrm{~h}$

\section{Liquefação}

$1 \mathrm{~h}$

Figura 2 - Descrição dos experimentos.

As batatas foram adquiridas no mercado local de Porto Alegre/RS, sendo de polpa e casca brancas. A concentração batata:água utilizada foi de 2:1. O pH foi controlado por meio de solução tampão de ácido cítrico e citrato de sódio. Os experimentos foram realizados em erlenmeyers de $250 \mathrm{~mL}$ com conteúdo de mosto de $200 \mathrm{~g}$. Para o controle de contaminação foram empregados $10 \mathrm{~mL}$ 
de solução do antibiótico doxiciclina na concentração 3,4 g/L. Todos os experimentos foram realizados em shaker a ar sob a agitação de $175 \mathrm{rpm}$, com exceção da etapa onde ocorreu aquecimento a $85^{\circ} \mathrm{C}$, neste caso o aquecimento foi realizado em banho-maria com agitações manuais a cada 5 min.

As médias dos resultados foram comparadas segundo teste de Tukey.

\subsection{Estudo sobre o efeito do micro-ondas na hidrólise}

Os resultados do estudo descrito na seção 2.2 foram parâmetros para a definição dos experimentos com o micro-ondas. Portanto, as justificativas para o estabelecimento das condições experimentais são discutidas na apresentação dos resultados (Seção 3). A metodologia é equivalente a já apresentada, porém, nestes experimentos, foi adicionada uma etapa anterior à liquefação, que consistiu no aquecimento prévio em micro-ondas doméstico. Nestes experimentos optou-se por reduzir a concentração da batata para 1,5:1, objetivando tornar o meio mais fluido. Também foram realizados experimentos de fermentação, utilizando $0,0175 \mathrm{~g} / \mathrm{g}$ de batata da levedura Saccharomyces cerevisiae, cepa CAT-1 produzida pela LNF.

\section{RESULTADOS}

$\mathrm{Na}$ etapa de caracterização da batata obteve-se o teor de umidade $67,83 \%$, glicose $2,24 \%$, frutose $1,41 \%$ e glicose total $21,09 \%$.

Os resultados do estudo sobre o efeito da temperatura de liquefação na hidrólise são apresentados na Tabela 1 (experimentos realizados em triplicata).

Tabela 1 - Efeito da temperatura de liquefação na hidrólise.

\begin{tabular}{|c|c|c|c|c|}
\hline Experimento & $\begin{array}{l}\text { Temperatura de } \\
\text { liquefação }\left({ }^{\circ} \mathrm{C}\right)\end{array}$ & Enzima & $\begin{array}{c}\text { Concentração média } \\
\text { de Glicose* (g/L) }\end{array}$ & Desvio padrão \\
\hline 1 & 85 & Spezyme RSL & $173,24^{\mathrm{a}}$ & 4,63 \\
\hline 2 & 70 & Spezyme RSL & $70,99^{\mathrm{b}}$ & 3,07 \\
\hline 3 & 70 & Spezyme RSL + GC 626 & $68,08^{b}$ & 2,53 \\
\hline 4 & 60 & GC 626 & $42,04^{\mathrm{c}}$ & 1,08 \\
\hline
\end{tabular}

*As médias que não compartilham a mesma letra são significativamente diferentes, segundo o teste de Tukey com 95\% de confiança. Utilizou-se a concentração de batata 2:1.

Considerando os dados da caracterização da batata e a concentração da mesma no mosto, o resultado teórico esperado na hidrólise era de $169,84 \pm 10,76 \mathrm{~g} / \mathrm{L}$, o que indica que, apenas no experimento 1 , o resultado máximo foi alcançado.

Segundo Srichuwong et al. (2012), em geral a gelatinização do amido de batata-doce ocorre entre $60 \mathrm{a} 85^{\circ} \mathrm{C}$. O melhor resultado obtido na temperatura de $85^{\circ} \mathrm{C}$ provavelmente se deve à completa gelatinização do amido. Os ensaios a $85^{\circ} \mathrm{C}$ apresentaram diferença visível em relação aos demais, ocorrendo um maior escurecimento, grânulos mais entumecidos e vítreos, que são características físicas da gelatinização. 


\section{9 a 22 de outubro de 2014 \\ Florianópolis/SC}

No que se refere à comparação do desempenho das duas enzimas de liquefação testadas, os resultados não são muito conclusivos. Avaliando os resultados dos experimentos 2 e 3 aparentemente não há diferença, mas, para uma afirmação mais correta, seriam necessários mais experimentos ampliando a abrangência das variáveis de estudo.

Deu-se sequência ao estudo, utilizando micro-ondas para pré-aquecer os meios até a temperatura $85^{\circ} \mathrm{C}$, já que esta temperatura se demonstrou importante para uma boa hidrólise. Optou-se por utilizar apenas a Spezyme RSL neste estudo, que foi adicionada logo após o pré-aquecimento com micro-ondas. Além da hidrólise foi realizada fermentação, iniciada simultaneamente com a sacarificação. Foram realizados três experimentos com quatro repetições, o primeiro experimento consistiu no método convencional de aquecimento a $85^{\circ} \mathrm{C}$ em banho-maria, para fins de comparação. O segundo experimento consistiu no aquecimento prévio em micro-ondas, seguido por liquefação em agitador orbital a $70^{\circ} \mathrm{C}$. O terceiro experimento foi realizado da mesma forma, mas em agitador orbital a $60^{\circ} \mathrm{C}$. Nos ensaios com micro-ondas a Pectinex Ultra AFP não foi adicionada, pois se desejou avaliar se o método com micro-ondas também implicaria em resultados positivos sobre a redução da viscosidade. Os resultados destes experimentos são apresentados na Tabela 2.

Tabela 2 - Produção de etanol em diferentes condições de liquefação e SSF.

\begin{tabular}{|c|c|c|c|}
\hline \multirow{2}{*}{ Experimento } & \multirow{2}{*}{ Resumo } & \multicolumn{2}{|c|}{ Etanol $\left(\%,{\left.\mathrm{v} \cdot \mathrm{v}^{-1}\right)^{*}}^{*}\right.$} \\
\cline { 2 - 4 } & Convencional, com a Pectinex Ultra AFP & $24 \mathrm{~h}$ & $48 \mathrm{~h}$ \\
\hline 1 & Micro-ondas, shaker a $70^{\circ} \mathrm{C}$, sem a Pectinex Ultra AFP & $9,82^{\mathrm{a}}$ & $10,72^{\mathrm{a}}$ \\
\hline 2 & Micro-ondas, shaker a $60^{\circ} \mathrm{C}$, sem a Pectinex Ultra AFP & $9,63^{\mathrm{b}, \mathrm{c}}$ & $10,4^{\mathrm{a}, \mathrm{b}}$ \\
\hline 3 & $10,4^{\mathrm{a}, \mathrm{b}}$ \\
\hline
\end{tabular}

*As médias que não compartilham a mesma letra são significativamente diferentes, segundo o teste de Tukey com 95\% de confiança. Utilizou-se a concentração de batata 1,5:1.

Com base nos resultados da caracterização da batata, o valor teórico máximo de etanol foi calculado como 11,13\%. Considerando o menor e o maior valor experimental apresentados na Tabela 2, a eficiência de conversão variou de 85 a 97\%, o que indica que os resultados foram satisfatórios.

Não foi possível notar efeito isolado do micro-ondas sobre a viscosidade. Desta forma, conclui-se que este método não substitui o efeito da Pectinex Ultra AFP, como pode ser observado na imagem comparativa da Figura 3.

Em 24h de fermentação a produção de etanol no experimento 1 foi um pouco mais elevada em relação às demais, talvez pela a presença da Pectinex Ultra AFP, que pode ter implicado em uma breve aceleração do processo pela redução da viscosidade. Mas, após $48 \mathrm{~h}$ de fermentação, os resultados se equipararam, não havendo diferença significativa entre eles segundo a comparação das médias pelo método de Tukey.

Os resultados apresentados na Tabela 2 indicam que o uso do micro-ondas permite a redução da temperatura na liquefação. Inclusive, é possível que a temperatura possa ser reduzida abaixo dos $60{ }^{\circ} \mathrm{C}$, havendo a necessidade de mais experimentos para confirmar tal hipótese. 


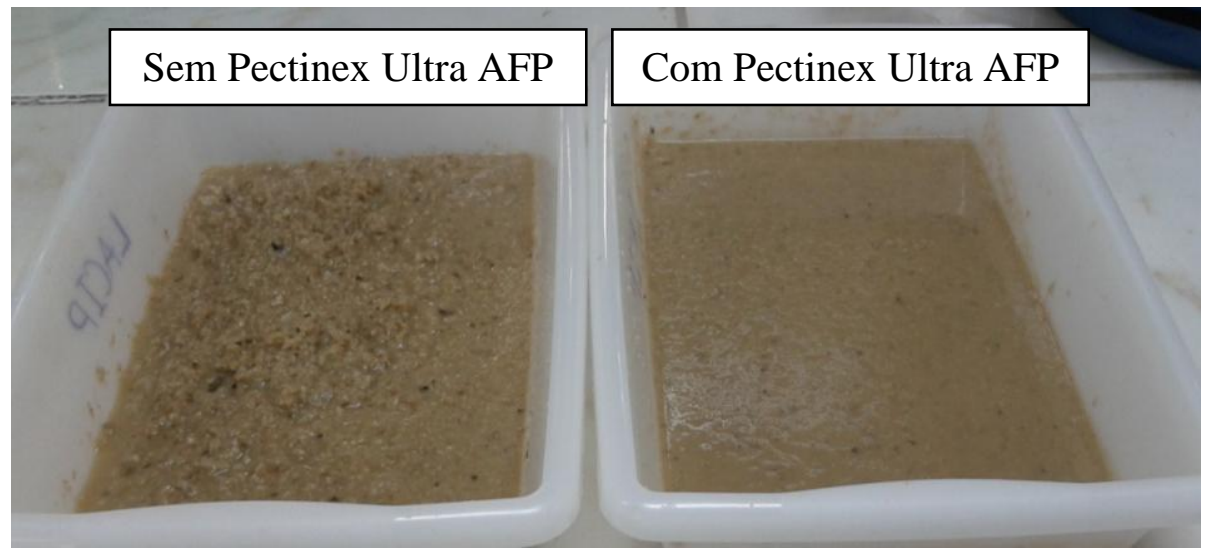

Figura 3 - Imagem comparativa de experimentos com e sem a Pectinex Ultra AFP.

Diante do exposto, o acréscimo do micro-ondas ao método é uma opção a ser considerada, quando se deseja reduzir o custo energético, pois o tempo de aquecimento necessário neste caso foi de 2,5 min por erlenmeyer, além da temperatura de liquefação poder ser reduzida, de $85^{\circ} \mathrm{C}$ para $60^{\circ} \mathrm{C}$.

$\mathrm{Na}$ literatura foram encontrados poucos trabalhos semelhantes (inferior a 20), sendo ainda a maioria referente à utilização de micro-ondas na hidrólise ácida do amido. Entre eles, chama-se a atenção para os de Saifuddin e Hussain (2011), Zhang et al. (2012) e Zhang et al. (2011), onde foi avaliado o efeito das micro-ondas sobre as enzimas amilolíticas, em todos os trabalhos há o relato de que o desempenho da atividade enzimática foi potencializado. Os resultados de Saifuddin e Hussain (2011) demonstraram que a hidrólise enzimática do amido de sagu combinada com as micro-ondas aumentou a taxa de reação inicial duas vezes. Segundo os autores, os resultados indicam que há uma ativação específica não térmica sobre as enzimas causada pelas micro-ondas. Estas informações coletadas na literatura são de grande contribuição, demonstram que também é interessante a realização de experimentos de aquecimento com o micro-ondas simultâneo com o processo de liquefação.

\section{CONCLUSÃO}

Para uma hidrólise adequada é necessário que a estrutura granular do amido seja degradada, tornando-o mais vulnerável ao ataque enzimático. Para tanto, uma dada quantidade de energia deve ser empregada. Nos ensaios aplicando o método convencional de aquecimento, a temperatura de $85^{\circ} \mathrm{C}$ foi identificada como um ponto que caracteriza tal energia. $\mathrm{O}$ método com micro-ondas mostrou-se bastante promissor, possibilitando a redução da temperatura de liquefação para $60^{\circ} \mathrm{C}$. Esta simplificação pode estar associada à maior desintegração do amido pelo efeito da energia eletromagnética. É possível que este processo permita ainda mais a redução da temperatura de liquefação, o que deve ser constatado mediante a realização de mais experimentos, inclusive com a enzima de liquefação presente durante o aquecimento.

Acredita-se que o emprego do micro-ondas seja uma possibilidade factível na produção industrial de etanol. Certamente, ainda seria necessária uma adaptação da tecnologia para a maior 
escala. Porém, trabalhos como o apresentado servem como fomento para a consolidação do microondas no campo industrial.

\section{REFERÊNCIAS}

FIGUEIREDO, E. Métodos físico-químicos para a análise de alimentos. São Paulo: Instituto Adolfo Lutz, 2008.

MASIERO, S. S. Microusinas de etanol de batata-doce: viabilidade econômica e técnica. Porto Alegre: Dissertação de mestrado em Engenharia Química, Universidade Federal do Rio Grande do Sul (UFRGS), 2012.

RISSO, R. S. Etanol de Batata-doce: Otimização do Pré-processamento da Matéria-prima e da Hidrólise Enzimática. Porto Alegre: Dissertação de mestrado em engenharia química, Universidade Federal do Rio Grande do Sul (UFRGS), 2014.

SAIFUDDIN, N; HUSSAIN, R.. Microwave Assisted Bioethanol Production from Sago Starch by Co-Culturing of Ragi Tapai and Saccharomyces Cerevisiae. Journal of Mathematics and Statistics, p. 198-206, 2011.

SRICHUWONG, S; ORIKASA, T.; MATSUKI, J; SHIINA, T.; KOBAYASHI, T.; TOKUYASU, K. Sweet potato having a low temperature-gelatinizing starch as a promising feedstock for bioethanol production. Biomass and Bioenergy, v. 39, p. 120 - 127, 2012.

ÚNICA. UNICADATA. Disponível em: < http://www.unicadata.com.br/ >. Acesso em: 04 jan 2014.

ZHANG, X.; CAO, T.; TIAN, X; GAI, D. Effect of microwave irradiation on the structure of glucoamylase. Process Biochemistry, v. 47, p. 2323-2328, 2012.

ZHANG, X.-y.; QIN, W.-q.; TIAN, X.-d.; HUANG, M.. Effect of microwave irradiation on secondary structure of $\alpha$-amylase by circular dichroism. J. Cent. South Univ. Technol., v. 18, p. 1029-1033, 2011. 